\title{
Glycosylation of Anti-Thyroglobulin IgG1 and IgG4 Subclasses in Thyroid Diseases
}

\author{
Yuan Li Chenxu Zhao a,b Keli Zhao ${ }^{c, d}$ Nan Yu ${ }^{a}$ Yan Lic, d Yang Yu \\ Yang Zhang $^{a}$ Zhijing Song ${ }^{c, d}$ Youyuan Huang ${ }^{a}$ Guizhi Lu ${ }^{a}$ Ying Gao ${ }^{a}$ \\ Junqing Zhang ${ }^{\text {a }}$ Xiaohui Guo $^{\text {a }}$ \\ ${ }^{a}$ Department of Endocrinology, Peking University First Hospital, Beijing, China; ${ }^{b}$ Department of Endocrinology, \\ The First Hospital of Hebei Medical University, Shijiazhuang, China; ' Institute of Biophysics, Chinese Academy of \\ Sciences, Beijing, China; ${ }^{\mathrm{d} C o l l e g e}$ of Life Science, University of the Chinese Academy of Sciences, Beijing, China
}

\section{Keywords}

Thyroglobulin antibody · lgG subclasses · Glycosylation ·

Thyroid diseases

\begin{abstract}
Objective: Thyroglobulin antibodies (TgAb), principally comprising immunoglobulin $\mathrm{G}(\mathrm{lgG})$, are frequently found in healthy individuals. Previously, we showed that the glycosylation levels of $\mathrm{TgAb} \lg \mathrm{G}$ differed across various thyroid diseases, suggesting an important role of glycosylation on antibodies in the pathogenesis of thyroid diseases. Since lgG1 and IgG4 are the primary $\mathrm{TgAb} \operatorname{lgG}$ subclasses, this study aimed to investigate the glycosylation of $\mathrm{TgAb} \operatorname{lgG} 1$ and IgG4 subclasses in thyroid diseases. Methods: TgAb IgG was purified by affinity chromatography from the serum of patients with Hashimoto's thyroiditis (HT) $(n=16)$, Graves' disease (GD) $(n=8)$, papillary thyroid carcinoma (PTC) $(n=6)$, and PTC with histological lymphocytic thyroiditis (PTC-T) $(n=9)$ as well as healthy donors $(n=10)$. TgAb $\operatorname{lgG} 1$ and $\lg \mathrm{G} 4$ concentrations were determined by enzyme-linked immunosorbent assay, and a lectin microassay was used to assess TgAb lgG1 and lgG4 glycosylation. Results: Significantly elevated mannose, sialic acid, and galactose levels on TgAb IgG1 were found in HT and PTC patients compared to GD
\end{abstract}

patients and healthy controls (all $p<0.05$ ). The mannose, sialic acid, and core fucose levels on TgAb lgG1 in PTC-T patients were higher than in healthy controls (all $p<0.05$ ). Additionally, TgAb IgG1 from PTC-T patients exhibited lower sialylation than that from patients with PTC and higher fucosylation than that from patients with HT (both $p<0.05$ ). However, TgAb IgG4 glycosylation did not differ among the five groups ( $p<0.05$ ). Conclusion: Our study describes different distributions of $\mathrm{TgAb} \lg \mathrm{G} 1$ glycosylation in various thyroid diseases. The aberrantly increased glycosylation levels of TgAb IgG1 observed in HT, PTC, and PTC-T might be indicative of immune disorders and participate in the pathogenesis of these diseases.

() 2020 European Thyroid Association Published by S. Karger AG, Basel

\section{Introduction}

Thyroglobulin antibodies (TgAb), frequently found in healthy individuals, are serological markers of both Hashimoto's thyroiditis (HT) and Graves' disease (GD)

Yuan Li and Chenxu Zhao are co-first authors and contributed equally to the article. 
[1]. In papillary thyroid carcinoma (PTC), the rate of $\mathrm{TgAb}$ positivity is around $20 \%$, approximately two-fold greater than that among the general population [2]. The increased presence of PTC in patients with HT reflects a relationship between the pathogenesis of PTC and that of HT [3-8].

$\mathrm{TgAb}$ are principally composed of immunoglobulin $\mathrm{G}$ (IgG) and glycoprotein, and certain changes in the immunological characteristics of IgG, such as the distribution of subclasses and glycosylation levels, have been identified to greatly influence antibody functions and to be related to immune responses in various diseases $[9$, 10]. Furthermore, $\operatorname{TgAb} \operatorname{IgG}$ has been confirmed in vitro to mediate antibody-dependent cell-mediated cytotoxicity (ADCC) [11] and are involved in the pathogenesis of HT, GD, and PTC with histological lymphocytic thyroiditis (PTC-T). Additionally, the presence of TgAb is an individual risk factor for PTC and might be associated with the thyroglobulin (Tg) clearance in PTC [12-14].

The glycans of IgG are attached to the asparagine residue on both fragment crystallizable ( $\mathrm{Fc}$ ) (approximately $80-90 \%$ of total IgG N-glycosylation) and fragment antigen-binding (Fab) regions. Classical IgG N-glycans consist of a dual-antenna structure that is variously modified by bisecting $\mathrm{N}$-acetylglucosamine (GlcNAc), a core fucose, and terminal glycosyls (sialic acid and galactose) $[15,16]$ (online suppl. Fig. S1; for all online suppl. material, see www.karger.com/doi/10.1159/000507699). It has been observed that the glycosylation of IgG has important implications for its biological efficacy $[17,18]$. The removal of glycans on the Fc region of IgG resulted in reduced affinity for $\mathrm{Fc} \gamma \mathrm{R}$ and thereby failure to mediate ADCC [19-22], and the Fab N-glycans were demonstrated to influence the antigen-binding ability and half-life of $\operatorname{IgG}[23,24]$. Alterations in IgG glycosylation profiles have been reported for many autoimmune diseases and cancers $[17,25,26]$. For example, we recently demonstrated that the glycosylation level of TgAb IgG in HT patients was higher than that in healthy individuals [27], and in another study, we reported that TgAb IgG glycosylation differed in serum from patients with HT, GD, and PTC [28]. These findings indicate that aberrant glycosylation of $\mathrm{TgAb}$ IgG might reflect the immunological and pathological processes of thyroid diseases and contribute to disease pathogenesis by modulating immune responses.

In addition to IgG glycosylation, the subclass distribution of IgG is also associated with its effector functions. Human IgG can be divided into four IgG subclasses IgG1, IgG2, IgG3, and IgG4 [29] - each of which has dis- tinct effector functions due to different affinities for $\mathrm{F} c \gamma \mathrm{R}$ $[30,31]$. Aberrant glycosylation among IgG subclasses has been observed in autoimmune diseases [32-35]. The levels of galactosylation, sialylation, and bisecting GlcNAc on both IgG1 and IgG2 subclasses have been reported to be decreased in granulomatosis with polyangiitis. In rheumatoid arthritis, a similar galactosylation defect was detected for IgG1, IgG2, and IgG4, but not for IgG3 [32]. Therefore, both the subclass distribution and glycosylation of IgG reflect the human pathological states. Changes in IgG subclass glycosylation are speculated to be associated with the role of $\mathrm{TgAb}$ in thyroid diseases. However, to our knowledge, subclass-specific alterations in $\mathrm{TgAb} \operatorname{IgG}$ glycosylation have not been reported.

In this study, we employed a high-density lectin microarray to examine the glycosylation levels of IgG1 and IgG4 subclasses of TgAb in serum from HT, GD, PTC, and PTC-T patients and healthy controls. We further investigated differences in glycosylation of $\mathrm{TgAb}$ IgG subclasses among various thyroid diseases to provide new insight into the role of $\mathrm{TgAb}$ in these diseases.

\section{Subjects and Methods}

\section{Subjects}

Thirty-nine serum samples were collected from patients with thyroid diseases diagnosed at Peking University First Hospital from 2012 to 2017 . The patient population comprised the following four groups: HT patients $(n=16)$, GD patients $(n=8)$, PTC patients $(n=6)$, and PTC-T patients $(n=9)$. Serological identification showed TgAb positivity for all patients. HT patients were all previously diagnosed based on fine needle aspiration cytology, negative TSH receptor antibodies, with or without thyroid peroxidase antibodies (TPOAb). GD was diagnosed by cytology on fine needle aspiration cytology specimens, and the diagnosis of PTC was based on thyroidectomy specimens. When PTC was associated with the typical signs of HT in the surrounding areas, including a dense lymphoplasmacytic infiltrate with destruction of follicular cells, extensive Hürthle cell change, and pronounced germinal center formation, it was defined as PTC-T. In all groups there was absence of other types of thyroid diseases. The control group consisted of 10 healthy individuals matched for age and sex. The healthy controls were euthyroid, negative for serum $\mathrm{TgAb}$ and $\mathrm{TPOAb}$, and without abnormal ultrasonic thyroid manifestations; there were no relevant medical or family histories of thyroid diseases either. None of the participants had infectious diseases, including human immunodeficiency virus and hepatitis B virus infection, or other autoimmune diseases, including rheumatoid arthritis, multiple sclerosis, type 1 diabetes mellitus, and systemic lupus erythematosus. In addition, there was no evidence of inherited or acquired variation in the thyroxine-binding globulin concentration in all subjects. Serum TgAb and TPOAb were analyzed using a Cobas e601 (Roche Diagnostics, Basel, Switzerland) with 
Table 1. Demographic data and TgAb levels in the HT, GD, PTC, PTC-T, and CON groups

\begin{tabular}{|c|c|c|c|c|c|}
\hline & $\mathrm{HT}(n=16)$ & $\mathrm{GD}(n=8)$ & PTC $(n=6)$ & PTC-T $(n=9)$ & $\mathrm{CON}(n=10)$ \\
\hline Age, years & $37.1 \pm 12.4$ & $39.9 \pm 18.8$ & $42.0 \pm 10.7$ & $44.4 \pm 12.7$ & $38.3 \pm 16.0$ \\
\hline Sex, f/m & $15 / 1$ & $5 / 3$ & $5 / 1$ & $9 / 0$ & $9 / 1$ \\
\hline
\end{tabular}

CON, healthy controls; GD, Graves' disease; HT, Hashimoto's thyroiditis; PTC, papillary thyroid carcinoma; PTC-T, papillary thyroid carcinoma with histological lymphocytic thyroiditis; TgAb, thyroglobulin antibodies.

an electrochemiluminescence immunoassay (reference ranges 0-115 IU/mL for TgAb and $0-34 \mathrm{IU} / \mathrm{mL}$ for TPOAb). All samples were stored at $-80^{\circ} \mathrm{C}$ until use.

\section{Purification of Serum $\operatorname{IgG}$}

IgG purification was performed according to the manufacturer's instructions using a Hitrap Protein G HP (5 mL) column (GE Healthcare, Eindhoven, The Netherlands) installed on an AKTA purifier. Serum samples were first filtered through $0.20-\mu \mathrm{m}$ Minisart filters (Merck Millipore, Bedford, MA, USA), diluted 1:5 with binding buffer (0.02 $\mathrm{M}$ Tris, $\mathrm{pH} 7.2)$ to maintain $\mathrm{pH}$ and injected onto the Hitrap Protein G HP column. After removing unbound protein by washing with 5 column volumes of binding buffer, the bound IgG was eluted with elution buffer ( $0.1 \mathrm{M}$ glycine, $\mathrm{pH}$ 2.7). The $\mathrm{pH}$ of the IgG solution was adjusted to 7.4 using neutralization buffer ( $0.2 \mathrm{M}$ Tris, $\mathrm{pH} 9.0)$, and the sample was ultrafiltered and exchanged into phosphate-buffered saline (PBS) $(\mathrm{pH}$ 7.4) using Ultra Centrifugal Filters (Merck KGaA, Darmstadt, Germany).

\section{Purification of $\operatorname{TgAb} \operatorname{IgG}$}

Affinity purification for TgAb IgG was performed using a Tg affinity column as described in the literature [27]. Briefly, $5 \mathrm{mg}$ of human Tg (Merck Millipore) was bound to cyanogen bromideactivated Sepharose 4B (5 mL; Sigma-Aldrich, Saint Louis, MO, USA) in coupling buffer ( $0.1 \mathrm{M}$ sodium bicarbonate buffer containing $0.5 \mathrm{M}$ sodium chloride, $\mathrm{pH} 8.3$ ). After removing unbound $\mathrm{Tg}$, the Sepharose gel-bound $\mathrm{Tg}$ was transferred to an XK16/20 column with two adapters (GE Healthcare) for TgAb IgG purification, similar to the procedure for IgG purification, using $0.01 \mathrm{M}$ PBS ( $\mathrm{pH} 7.4$ ) for binding buffer and $0.1 \mathrm{M}$ glycine containing $0.5 \mathrm{M}$ sodium chloride $(\mathrm{pH}$ 2.7) for elution. Concentrations of $\mathrm{TgAb}$ IgG were assessed using the bicinchoninic acid protein assay. All TgAb IgG samples were frozen at $-80^{\circ} \mathrm{C}$ until use.

Determination of IgG1 and IgG4 Concentrations in TgAb IgG Samples Using Enzyme-Linked Immunosorbent Assay

The double antibody enzyme-linked immunosorbent assay (ELISA) method was employed to evaluate the relative content of IgG1 in TgAb IgG samples from the HT, GD, PTC, PTC-T, and healthy control groups. The commercial human IgG1 (Abcam, Cambridge, UK) was used as standard. Mouse anti-human IgG1 monoclonal antibody $(10 \mu \mathrm{g} / \mathrm{mL}$; clone HP6001; SouthernBiotech, Birmingham, AL, USA) was coated onto polystyrene microtiter plates (Costar, Cambridge, MA, USA). The TgAb IgG samples (diluted 1:50-1:1,000 with PBS) and IgG1 standards with serial con- centrations were added and incubated for $1 \mathrm{~h}$ at $37^{\circ} \mathrm{C}$. A horseradish peroxidase-labeled mouse anti-human IgG monoclonal antibody (1:2,000; clone H2; Abcam) was used to detect bound IgG1. The plates were washed three times with PBS containing $0.05 \%$ Tween-20 (PBST) after each incubation step. The substrate solution consisted of $4 \mathrm{mg} / \mathrm{mL}$ o-phenylenediamine in $0.05 \mathrm{M}$ sodium citrate, and $0.003 \%$ freshly added $\mathrm{H}_{2} \mathrm{O}_{2}$ was applied to each plate for color development. The reaction was stopped with $1 \mathrm{M}$ hydrochloric acid. Absorbance at $490 \mathrm{~nm}$ was recorded. The concentration of IgG1 in each sample was derived from the standard curve generated using IgG1 standards (online suppl. Fig. S2A).

The concentrations of IgG4 in TgAb IgG samples were evaluated using an ELISA similar to that used for TgAb IgG1. The commercial human IgG4 (Abcam) was used as standard. Plates were coated with a mouse anti-human IgG4 monoclonal antibody $(10 \mu \mathrm{g} / \mathrm{mL}$; clone HP6025; SouthernBiotech). Concentrations of IgG4 in TgAb IgG samples were calculated from the IgG4 standard curve (online suppl. Fig. S2B).

After appropriate predilution with PBST, the final concentration of both the IgG1 and IgG4 in all TgAb IgG samples was adjusted to the same level (the original concentrations of IgG1 and IgG4 in TgAb samples are shown in online suppl. Table S1), then the samples were applied for subsequent lectin microarray detection.

\section{Glycosylation of TgAb IgG1 and IgG4 Based on High-Density Lectin Microarray}

The commercial lectin microarray chip used in this study consists of triplicate spots of 56 lectins (see online suppl. Table S2 for the list of lectins) (Bio-Technology Co. Ltd., Guangzhou, China). The microarray was blocked with $3 \%$ bovine serum albumin diluted in $0.05 \mathrm{M}$ ethanolamine buffer $(\mathrm{pH} 8.0)$ for $1 \mathrm{~h}$. The slide was then washed three times with PBST and $\mathrm{ddH}_{2} \mathrm{O}$ and dried by centrifugation at $500 \mathrm{~g}$ for $5 \mathrm{~min}$. After adjusting the IgG1 concentration of purified TgAb IgG from each subject to $20 \mu \mathrm{g} / \mathrm{mL}$ using PBST, 200- $\mu$ L TgAb IgG samples were applied to the microarray and incubated at room temperature (approximately $24-28^{\circ} \mathrm{C}$ ) for 2 h. An AF647-labeled mouse anti-human IgG1 antibody (clone $4 \mathrm{E} 3$; SouthernBiotech) was mixed with $0.02 \mathrm{M}$ sodium periodate at $4{ }^{\circ} \mathrm{C}$ for $1 \mathrm{~h}$ to oxidize the oligosaccharide chain attached to the antibody, after which $200 \mu \mathrm{L}$ of $2 \mu \mathrm{g} / \mathrm{mL}$ oxidized AF647-labeled mouse anti-human IgG1 antibody was hybridized to the microarray in sodium periodate at room temperature for $1 \mathrm{~h}$ in the dark. After washing three times with PBST, PBS, and deionized water, the array was dried by centrifugation at $500 \mathrm{~g}$ for $5 \mathrm{~min}$ and scanned using a Lux Scan 10K-A scanner (CapitalBio Corp., Beijing, China) 
Table 2. Detectable lectins and glycans on $\mathrm{TgAb} \operatorname{IgG} 1$ via lectin microarray

\begin{tabular}{rlll}
\hline & Abbreviation & Full name & Specificity \\
\hline 1 & PSA & Pisum sativum agglutinin & aMan, aGlc \\
2 & LCA & Lens culinaris agglutinin & a-D-mannose, $\alpha$-D-glucose \\
3 & AAL & Aleuria aurantia lectin & Fuca6GlcNAc \\
4 & LAL & Laburnum anagyroides lectin (gold chain) & best inhibitor is alpha-Me-L-fucose among monosaccharides \\
5 & SNA/EBL & Sambucus nigra lectin & Neu5Aca6Gal/GalNAc \\
6 & SNA-I & Sambucus nigra agglutinin I (elderberry bark) & alpha 2,6 linked sialic acid residues \\
7 & ConA & concanavalin A lectin & aMan, aGlc \\
8 & MNA-M & Morniga M lectin (black elderberry) & Man \\
9 & Black bean crude & Phaseolus vulgaris sp. lectin & GalNAc $>$ lactose $>$ galactose, sialic acid \\
10 & IRA & Iris hybrid lectin (Dutch iris) & N-acetyl-D-galactosamine \\
11 & HMA & Homarus americanus lectin (lobster) & aGalNAc, $\alpha$ Fuc, Neu5Ac \\
12 & RCAI/RCA120 & Ricinus communis agglutinin I & Gal \\
\hline
\end{tabular}

IgG, immunoglobulin G; TgAb, thyroglobulin antibody.

Table 3. Detectable lectins and glycans on TgAb IgG4 via lectin microarray

\begin{tabular}{|c|c|c|c|}
\hline 1 & PSA & Pisum sativum agglutinin & aMan, aGlc \\
\hline 2 & LCA & Lens culinaris agglutinin & $\alpha$-D-mannose, $\alpha$-D-glucose \\
\hline 4 & SNA-I & Sambucus nigra agglutinin I (elderberry bark) & alpha 2,6 linked sialic acid residues \\
\hline 5 & ConA & concanavalin A lectin & aMan, aGlc \\
\hline 6 & MNA-M & Morniga M lectin (black elderberry) & Man \\
\hline 9 & IRA & Iris hybrid lectin (Dutch iris) & $\mathrm{N}$-acetyl-D-galactosamine \\
\hline 10 & PHA-L & Phaseolus vulgaris leuco agglutinin & Gal $\beta 4 G l c N A c \beta 6($ GlcNAc $\beta 2$ Man $\alpha 3)$, Mana3 \\
\hline 11 & PHA-E & Phaseolus vulgaris erythro agglutinin & $\begin{array}{l}\text { Gal } \beta 4 \text { GlcNAc } \beta 2 \text { Man } \alpha 6(\text { GlcNAc } \beta 4) \\
(\text { GlcNAc } \beta 4 \text { Man } \alpha 3) \text { Man } \beta 4\end{array}$ \\
\hline
\end{tabular}

IgG, immunoglobulin G; TgAb, thyroglobulin antibody.

at a wavelength of $647 \mathrm{~nm}$. The image was subsequently converted to a digital format for analysis. The signal-to-noise $(\mathrm{S} / \mathrm{N})$ ratio (medium intensity of location prospects with respect to the background) of each lectin spot was calculated.

Glycosylation of IgG4 subclass in TgAb IgG samples was detected following the same protocol using the oxidized AF647-labeled mouse anti-human IgG4 antibody (clone HP6025; SouthernBiotech).

\section{Statistical Analysis}

Statistical analysis was carried out using SPSS 20.0. Quantitative data with a normal distribution are presented as mean \pm standard deviation; otherwise, median and quartiles are provided. Among the five groups, significant differences among continuous variables were assessed using one-way analysis of variance or the Kruskal-Wallis test. Differences between two groups were determined using the Student $t$ test (normal distribution data) or the Mann-Whitney U test (for nonnormal distribution data). A $p$ value $<0.05$ was considered statistically significant.

Glycosylation of TgAb IgG1 in Thyroid Disease

\section{Results}

\section{Demographic Data of Participants}

The study included $16 \mathrm{HT}$ patients, $8 \mathrm{GD}$ patients, 6 PTC patients, 9 PTC-T patients, and 10 healthy controls. As shown in Table 1, there was no significant difference in sex or age across the five groups $(p>0.05)$. However, the levels of TgAb in the HT, GD, PTC, and PTC-T groups were significantly higher than those in the healthy control group $(p<0.05)$.

\section{Detectable Lectins and Glycans of TgAb IgG1 and IgG4 by Lectin Microarray}

Glycosylation of TgAb IgG subclasses was evaluated by lectin microarray. To compare the glycan signals of TgAb IgG1 and IgG4, TgAb IgG samples were adjusted 


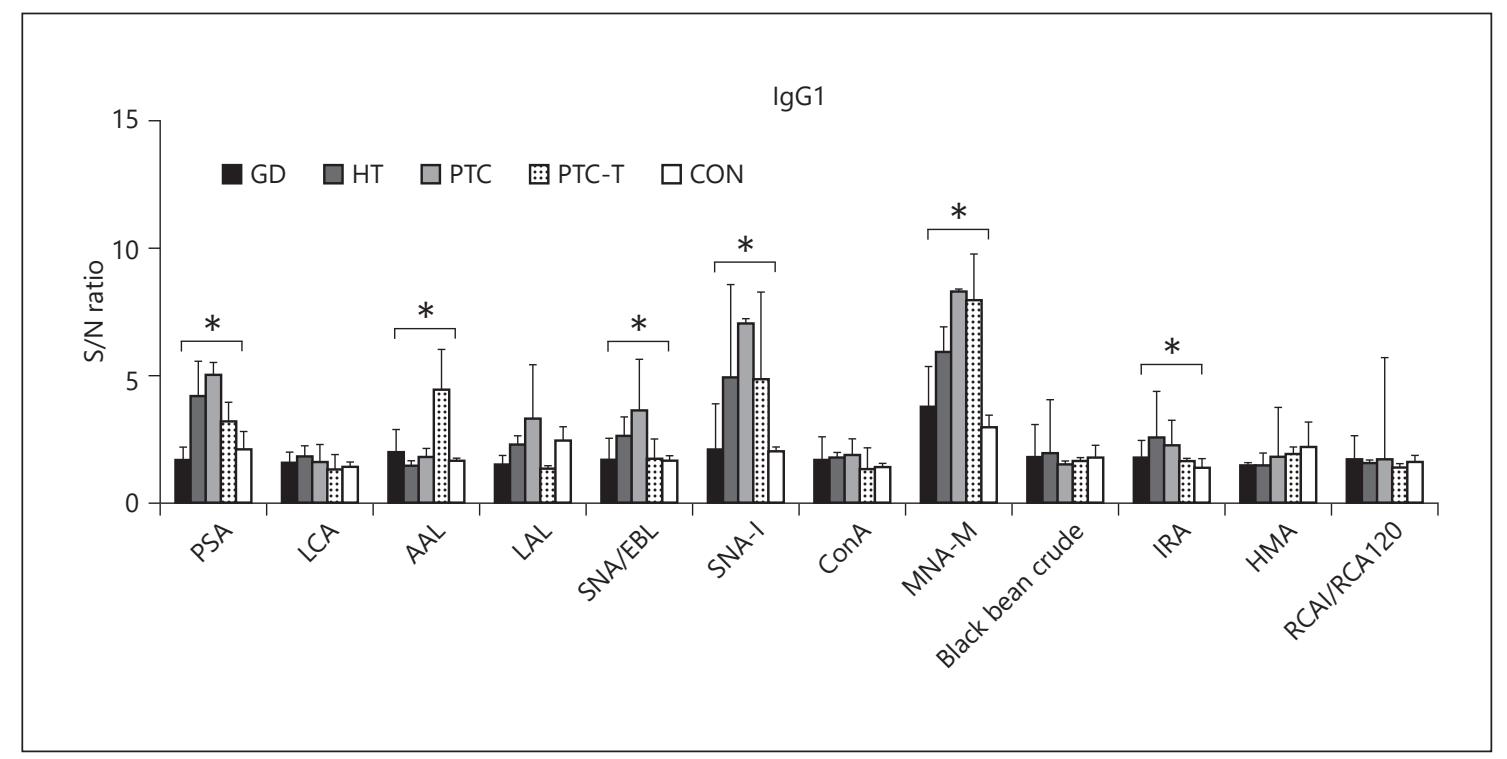

Fig. 1. Comparison of the $\mathrm{S} / \mathrm{N}$ ratios of twelve detectable lectins for TgAb IgG1 from the GD, HT, PTC, PTC-T, and CON groups. There were significant differences across the $\mathrm{S} / \mathrm{N}$ ratios of PSA, AAL, SNA/EBL, SNA-I, MNA-M, and IRA for TgAb IgG1 among the five groups. Binding specificities: PSA for mannose and GlcNAc; SNA/EBL for Neu5Aca6Gal; SNA-I for terminal sialic acid; MNA-M for mannose; IRA for galactose; and AAL for Fuca6GlcNAc. ${ }^{*} p<0.05$ among the five groups. AAL, Aleuria aurantia lectin; $\mathrm{CON}$, healthy controls; ConA, concanavalin A lectin; GD, Graves' disease; GlcNAc, N-acetylglucosamine; HMA, Homa-

to the same concentrations of IgG1 and IgG4; the signal of each lectin spot was represented by the $\mathrm{S} / \mathrm{N}$ ratio, and the cutoff was a value of $\mathrm{S} / \mathrm{N}$ ratio $\geq 1.2$. Lectins with detectable signals are defined as follows: (a) sample $\mathrm{S} / \mathrm{N}$ ratio - blank $\mathrm{S} / \mathrm{N}$ ratio $>0.5$; (b) (sample $\mathrm{S} / \mathrm{N}$ ratio) / (blank $\mathrm{S} / \mathrm{N}$ ratio) $>1.5$ for more than half of the samples of at least one group. There were 12 and 11 detectable lectins by microarray detection for $\operatorname{TgAb} \operatorname{IgG1}$ and IgG4, respectively (Tables 2, 3).

The Amounts of Glycan Present on TgAb IgG1 Differ among HT, GD, PTC, and PTC-T Patients and Healthy Controls

Comparative analysis of TgAb IgG1 glycosylation was performed, and the $\mathrm{S} / \mathrm{N}$ ratios of the following six lectins showed significant differences among the five groups: Pisum sativum agglutinin (PSA), Morniga M lectin (MNAM), Sambucus nigra agglutinin I (elderberry bark) (SNA-I), Sambucus nigra lectin (SNA/EBL), Iris hybrid lectin (Dutch iris) (IRA), and Aleuria aurantia lectin (AAL) (Fig. 1).

As shown in Figure 2, we found higher levels of mannose and/or GlcNAc, as indicated by PSA, on TgAb IgG1 rus americanus lectin (lobster); HT, Hashimoto's thyroiditis; IgG, immunoglobulin G; IRA, Iris hybrid lectin (Dutch iris); LAL, Laburnum anagyroides lectin (gold chain); LCA, Lens culinaris agglutinin; MNA-M, Morniga M lectin; PSA, Pisum sativum agglutinin; PTC, papillary thyroid carcinoma; PTC-T, papillary thyroid carcinoma with histological lymphocytic thyroiditis; RCAI/ RCA120, Ricinus communis agglutinin I; S/N, signal-to-noise; SNA/EBL, Sambucus nigra lectin; SNA-I, Sambucus nigra agglutinin I (elderberry bark); TgAb, thyroglobulin antibody.

from HT, PTC, and PTC-T patients than on TgAb IgG1 from GD patients and healthy controls $(p<0.05)$ (Fig. 2a). In HT patients, the amount of sialic acid attached to terminal galactose via $\alpha-2,6$ and $\alpha-2,3$ (Neu5Aca6Gal) on TgAb IgG1 detected by SNA/EBL was greater than that of healthy controls $(p<0.05)$; in PTC patients, the level of Neu5Aca6Gal on TgAb IgG1 was higher than those in healthy controls, GD, and PTC-T patients (all $p<0.05$ ) (Fig. 2b). Since the Neu5Aca6Gal oligosaccharide is the only modification of sialic acid on TgAb [27], the level can exactly reflect the level of terminal sialic acid. Compared to GD patients and healthy controls, increased terminal sialic acid on $\operatorname{TgAb} \operatorname{IgG} 1$ was found in patients with HT, PTC, and PTC-T (all $p<0.05$ ) (Fig. 2c). In patients with HT, PTC, and PTC-T, the mannose modification levels of $\mathrm{TgAb}$ IgG1 were significantly higher than those of healthy controls; there was a significantly lager amount of mannosylated TgAb IgG1 in PTC and PTC-T patients than in GD patients (all $p<0.05$ ) (Fig. 2d). IRA binds to galactose as well as to $\mathrm{N}$-acetyl-D-galactosamine [36]. Because glycans of human IgG lack $\mathrm{N}$-acetyl-D-galactosamine, the higher $\mathrm{S} / \mathrm{N}$ ratios for IRA lectins in $\mathrm{HT}$ and 

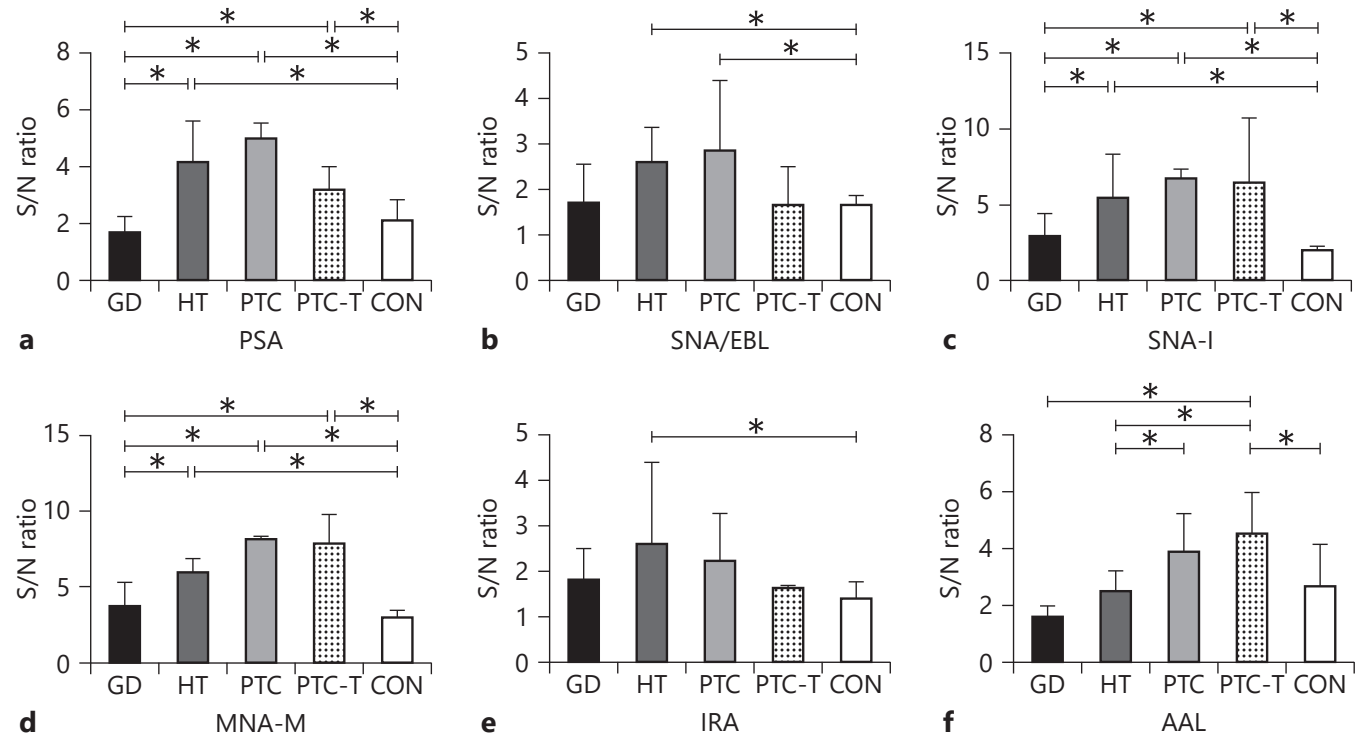

Fig. 2. Differences in the $S / N$ ratios of $T g A b$ IgG1 from the GD, HT, PTC, PTC-T, and CON groups. a PSA, specifically for mannose and GlcNAc, and the modification levels of mannose and/or GlcNAc on TgAb IgG1 were significantly higher in the HT, PTC, and PTC-T groups than in the GD and CON groups. b SNA/EBLbound Neu5Aca6Gal and the level of galactose-sialic acid were significantly higher in the HT group than in the CON group. In addition, the level of galactose-sialic acid was significantly higher in the PTC group than in the GD, PTC-T, and CON groups. c An increased level of sialic acid, which is recognized by SNA-I lectin, was found in the HT, PTC, and PTC-T groups compared to in the GD and CON groups. $\mathbf{d}$ MNA-M specifically recognizes mannose, and the mannose modification levels of TgAb IgG1 in the HT, PTC, and PTC-T groups were significantly increased compared with those in the CON group. In addition, the mannose modification levels of TgAb IgG1 in the PTC and PTC-T groups were significantly higher than those in the GD group. e An elevated level of galactose modification on $\operatorname{TgAb} \operatorname{IgG1}$, as indicated by increasing

PTC patients suggest that the $\operatorname{TgAb} \operatorname{IgG} 1$ galactose level was greater in these patients than that in healthy controls (all $p<0.05$ ) (Fig. 2e). Among the five groups, the level of core fucose on TgAb IgG1 in PTC patients was significantly higher than that in GD patients $(p<0.05)$ and tended to be higher than that of HT patients $(p=$ 0.077). TgAb IgG1 in PTC-T patients had significantly higher core fucose content than those in healthy controls, HT, and GD patients (all $p<0.05$ ) (Fig. 2f). No significant difference in glycosylation level of $\mathrm{TgAb}$ IgG1 was observed between the GD and heathy control groups $(p>0.05)$.

Glycosylation of TgAb IgG1 in Thyroid Disease
S/N ratios of IRA, was found in the HT and PTC groups compared with the CON group. f AAL binds Fuca6GlcNAc, and the modification level of core fucosylation of TgAb IgG1 was significantly higher in the PTC-T group than in the GD, HT, and CON groups. Additionally, the level of core fucose was significantly increased in the PTC group compared to the GD group. The PTC group exhibited a nonsignificant tendency toward an increased level of fucosylation of TgAb IgG1 compared with the HT group, but the difference was not significant $(p=0.077) .{ }^{*} p<0.05$. AAL, Aleuria aurantia lectin; CON, healthy controls; GD, Graves' disease; GlcNAc, N-acetylglucosamine; HT, Hashimoto's thyroiditis; IgG, immunoglobulin G; IRA, Iris hybrid lectin (Dutch iris); MNA-M, Morniga M lectin; PSA, Pisum sativum agglutinin; PTC, papillary thyroid carcinoma; PTC-T, papillary thyroid carcinoma with histological lymphocytic thyroiditis; S/N, signal-to-noise; SNA/EBL, Sambucus nigra lectin; SNA-I, Sambucus nigra agglutinin I (elderberry bark); $\operatorname{TgAb}$, thyroglobulin antibody.

The Amounts of Glycan Present on TgAb IgG4 Do Not Differ among HT, GD, PTC, and PTC-T Patients and Healthy Controls

According to our results, there was no significant differences in IgG4 glycosylation for $\mathrm{TgAb}$ IgG samples among the five groups $(p>0.05)$ (Fig. 3).

\section{Discussion}

Glycosylation is one of the most important posttranslational modifications of IgG, and it has been identified as essential for IgG-mediated effector functions [37, 38]. 


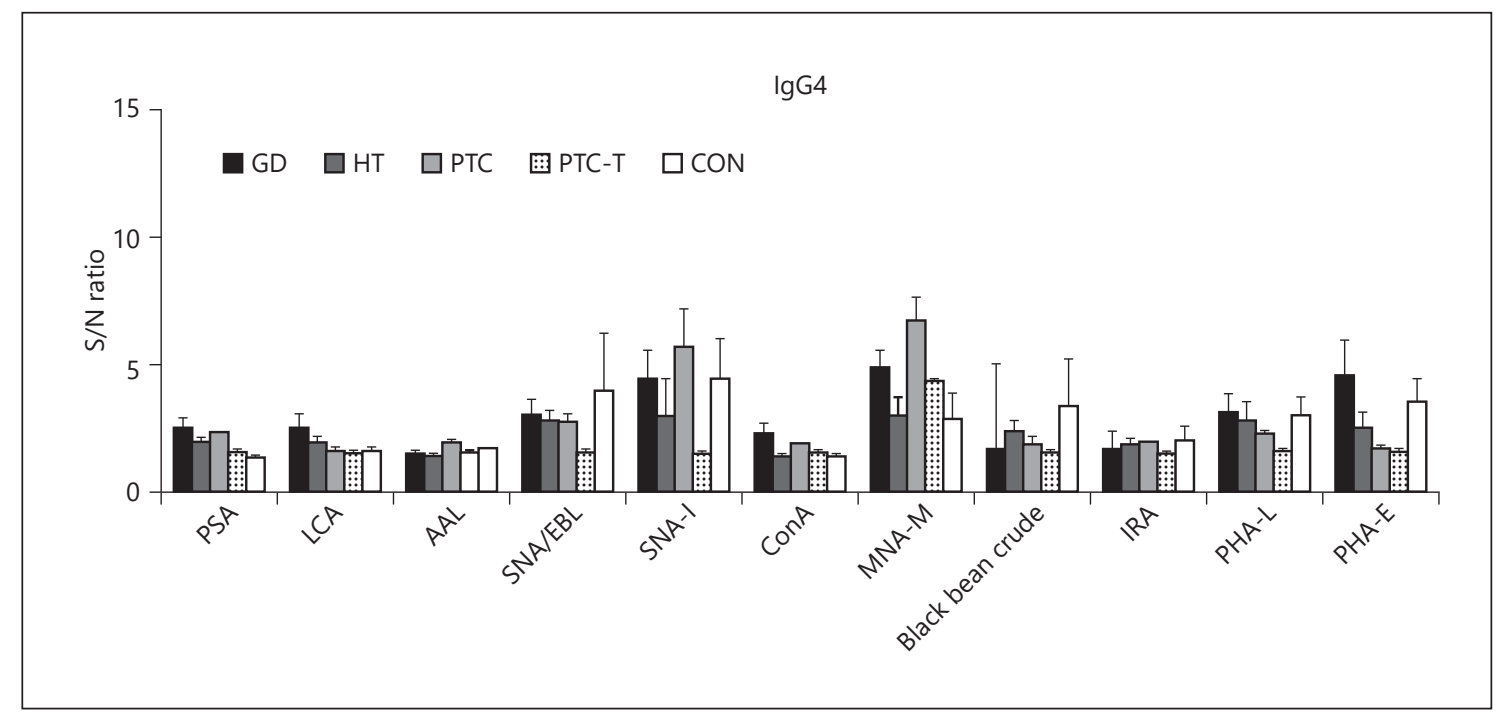

Fig. 3. Comparison of the $\mathrm{S} / \mathrm{N}$ ratios of eleven detectable lectins for TgAb IgG4 subclasses among the GD, HT, PTC, PTC-T, and CON groups. Among the five groups, no significant difference in the $\mathrm{S} / \mathrm{N}$ ratios of lectins for TgAb IgG4 was detected. AAL, Aleuria aurantia lectin; ConA, concanavalin A lectin; CON, healthy controls; GD, Graves' disease; HT, Hashimoto's thyroiditis; IgG, immunoglobulin G; IRA, Iris hybrid lectin (Dutch iris); LCA, Lens culinaris

Our recent work demonstrated that aberrant glycosylation of TgAb IgG in different thyroid diseases might be involved in their pathogenesis of thyroid diseases. Numerous other studies have shown that glycosylation of IgG varies across subclasses in autoimmune diseases [3235]. Accordingly, we assumed that changes in glycosylation of $\mathrm{TgAb}$ IgG might be subclass-specific due to the distinct biological characteristics of each IgG subclass. Moreover, altered IgG glycosylation might influence the IgG subclass distribution of $\mathrm{TgAb}$ in thyroid diseases. Thus, our evaluation of glycosylation levels on TgAb IgG subclasses will be helpful for further revealing the role of IgG glycosylation in various thyroid diseases.

In HT patients, TgAb IgG mainly consists of IgG1, IgG2, and IgG4 [29], whereas the dominant IgG subclasses in patients with GD, PTC, and nontoxic goiter are IgG1 and IgG4 [9]. Additionally, the IgG subclass distribution of TgAb IgG changed in the development of thyroid diseases [39]. Among these IgG subclasses, IgG1 exhibits the strongest ability to induce ADCC functions [40]. The association of high positivity rate and titer of TgAb IgG1 with hypothyroidism in HT suggests that TgAb IgG1 might contribute to the damage of thyroid follicular cells in $\mathrm{HT}$, which in turn affects the status of thyroid function [41]. Unlike IgG1, IgG4 is a poor activator of ADCC, and agglutinin; MNA-M, Morniga M lectin; PHA-E, Phaseolus vulgaris erythro agglutinin; PHA-L, Phaseolus vulgaris leuco agglutinin; PSA, Pisum sativum agglutinin; PTC, papillary thyroid carcinoma; PTC-T, papillary thyroid carcinoma with histological lymphocytic thyroiditis; S/N, signal-to-noise; SNA/EBL, Sambucus nigra lectin; SNA-I, Sambucus nigra agglutinin I (elderberry bark); TgAb, thyroglobulin antibody.

the high titers of $\operatorname{TgAb} \operatorname{IgG} 4$ detected in different thyroid diseases might represent prolonged antigenic stimulation [42]. Despite its dominance in HT patients, IgG2 is weak in mediating ADCC [43]. Considering that our serum samples were limited, we focused on the glycosylation of TgAb IgG1 and IgG4 in patients with HT, GD, PTC, and PTC-T as well as healthy controls.

Our results showed that the IgG1 subclass of TgAb displayed elevated glycosylation levels in HT, PTC, and PTC-T patients compared to GD patients and healthy controls, indicating that the mechanism underlying the glycosylation of $\operatorname{TgAb}$ IgG subclasses might vary across diverse thyroid diseases. The altered glycoforms of $\mathrm{TgAb}$ IgG1 between the HT and control groups were mostly similar to those of $\operatorname{TgAb} \operatorname{IgG}$, as reported in our previous study [28], though the unmodified glycosylation of $\mathrm{TgAb}$ IgG4 did not occur in parallel with the altered elevation in TgAb IgG glycosylation. This suggests that the changes in IgG1 subclass glycosylation, but not IgG4, are largely responsible for the differences in TgAb IgG glycosylation between HT patients and healthy individuals.

We observed greater amounts of mannose, sialic acid, core fucose, and galactose on TgAb IgG1 in HT patients compared to healthy controls, as well as a possible higher level of GlcNAc, as indicated by PSA. According to previ- 
ous publications, elevated levels of both mannose and galactose on IgG may enhance ADCC activity [44, 45], whereas high sialylation of IgG inhibits its binding with Fc $\gamma$ RIIIa and subsequently ADCC $[26,27]$. Removal of core fucose from IgG1 has been observed to be critical for enhancing ADCC [46, 47]. Given that the level of TgAb IgG1 glycosylation was quantified by lectin microarray in our study, the relative proportion of each mono- or oligosaccharide on $\operatorname{TgAb}$ IgG1 was not revealed in this study. Therefore, additional detailed studies are needed to illuminate the combined effects of altered glycan structures of TgAb IgG1 on ADCC and the pathogenesis of HT.

We further found that the levels of mannose, sialic acid, galactose, and core fucose on TgAb IgG1 in GD patients were identical to those of healthy individuals. Furthermore, the amounts of mannose and sialic acid on TgAb IgG1 in GD patients were significantly lower than those in HT patients. It seems that although both GD and HT are autoimmune thyroid diseases, the glycosylation level of $\operatorname{TgAb} \mathrm{IgG} 1$ is different between these two diseases. The central feature of HT is the infiltration of the thyroid by massive type 1 Thelper (Th1) lymphocytes, which secrete cytokines such as IFN- $\gamma$ and TNF- $\alpha$, leading to the destruction of thyrocytes $[48,49]$. By contrast, the thyroid-infiltrating lymphocytes in GD are mainly type $2 \mathrm{~T}$ helper (Th2) cells, which secrete cytokines such as IL-4, IL-5, and IL-6, finally increasing the antibody production of B cells and preventing apoptosis $[49,50]$. Galactosylation of IgG1 secreted by B cells was demonstrated to be upregulated by IFN- $\gamma$ [51]. The different glycosylation levels of TgAb IgG1 between GD and HT might result from the distinct cytokine profiles in each disease $[49,52$, 53]. IgG1 has the strongest ability of mediating ADCC, and different glycosylation levels of TgAb IgG1 between HT and GD may be related to the different functions of TgAb IgG1 in HT and GD. The glycosylation profiles of TgAb IgG1 in GD and HT may be used to distinguish these two disease processes.

Presence of positive serum TgAb in PTC was reported to be related with increased percentage of high-risk patients and extrathyroidal tumor extension [54], revealing an important role of $\mathrm{TgAb}$ in the pathogenesis of PTC. It has been reported that the serum IgG glycosylation profiles were altered in many kinds of cancers compared to nonmalignant controls [55-58]. In the present study, we found that levels of mannosylation, sialylation, and core fucosylation of TgAb IgG1 in PTC patients were markedly increased in comparison to those in healthy individuals. Additionally, the level of fucosylation of TgAb IgG1 in PTC patients tended to be increased compared to that

Glycosylation of TgAb IgG1 in Thyroid Disease in HT patients. It is believed that TgAb in PTC are mainly produced by $\mathrm{B}$ cells infiltrating into tumors [6]. Moreover, both Th1 and Th2 cytokines were expressed by thyroid-infiltrating lymphocytes of PTC patients $[59,60]$; the aberrant glycosylation of $\mathrm{TgAb} \operatorname{IgG} 1$ in PTC might also be associated with the immune disorders in the development of PTC. Enhanced expression of a1,6fucosyltransferase (FUT8), which is critical for the synthesis of core fucosylation [61], was detected in thyroids of PTC patients and related with disease progression [62]. Thus, the aberrant high level of core fucosylation of $\mathrm{TgAb}$ IgG1 in PTC patients might be a result of not only the immune factors, but also the overexpression of FUT8. Functional studies have shown that the glycan sites on Fc and Fab regions influence the binding of IgG to $\mathrm{F} c \gamma \mathrm{R}$ and antigens, respectively $[23,24]$. TgAb were demonstrated to accelerate the metabolic clearance of Tg in PTC [14]. Combined with our results, this indicates that the ability of TgAb IgG to clear Tg in PTC might be affected by glycosylation levels of TgAb IgG.

In PTC-T patients, the levels of mannose, sialic acid, and core fucose on TgAb IgG1 were significantly increased compared to those of healthy controls. The amounts of mannose and galactose of TgAb IgG1 from patients with PTC-T were also approximately equal to those from PTC and HT patients. However, TgAb IgG1 from patients with PTC-T exhibited lower sialylation than that from PTC patients and increased fucosylation compared to that of HT patients. A previous study revealed that the TgAb epitope pattern of PTC-T patients more closely resembled that of HT than PTC patients, suggesting that the regulation mechanisms involved in $\mathrm{TgAb}$ appearance in PTC-T might be more similar to those in HT [63]. Furthermore, it was shown earlier that both the infiltrated lymphocytes in the thyroid and the secreted cytokines in PTC-T were different from those in PTC or HT [60]. The glycosylation patterns of IgG were found to be regulated by many factors, such as stimuli of B cells, cytokine milieu, and extracellular glycosyltransferases $[51,64]$. It is speculated that the mechanism involved in the glycosylation of TgAb IgG1 in PTC-T might be a mixed immune response of both PTC and HT; meanwhile, it is distinct from those in PTC and HT alone.

In conclusion, the current study demonstrated that glycosylation changes on TgAb IgG among various thyroid diseases are subclass-specific. The IgG1 subclass of $\mathrm{TgAb}$ displayed elevated glycosylation levels in both HT, PTC, and PTC-T patients compared to GD patients and healthy controls, whereas no significant differences were found in the level of IgG4 subclass glycosylation among 
the five groups. We speculate that changes in IgG1 glycosylation predominate among the differences in TgAb IgG glycosylation, which might be involved in the pathogenesis of different thyroid diseases. Different glycosylation of TgAb IgG1 might influence the functions of TgAb in different thyroid diseases. Given that the regulation of IgG glycosylation is linked with multiple factors, additional studies regarding the mechanism of glycosylation on TgAb IgG subclasses are required for understanding the role of $\mathrm{TgAb}$ in various thyroid diseases and further supplementing the pathogenesis of thyroid diseases.

\section{Statement of Ethics}

This study was approved by the biomedical research ethics committee of the Peking University First Hospital, in compliance with the Declaration of Helsinki. All study participants gave written informed consent.

\section{Conflict of Interest Statement}

The authors declare that they have no conflict of interest.

\section{Funding Sources}

This work was supported by the National Natural Science Foundation of China (grant numbers 81370877 and 81770783).

\section{Author Contributions}

Concept and design: Ying Gao, Yan Li, Junqing Zhang, and Xiaohui Guo. Material preparation and data collection: Yuan Li, Chenxu Zhao, Keli Zhao, Nan Yu, Yan Li, Yang Yu, Yang Zhang, Zhijing Song, Youyuan Huang, and Guizhi Lu. Data analysis and investigation: Yuan Li, Chenxu Zhao, Keli Zhao, Zhijing Song, and Ying Gao. Drafting of the manuscript: Yuan Li and Chenxu Zhao. Revision of the manuscript: Ying Gao. Technical support: Youyuan Huang. Both co-first authors contributed equally to the article.

\section{References}

1 Piechaczyk M, Bouanani M, Salhi SL, Baldet L, Bastide M, Pau B, et al. Antigenic domains on the human thyroglobulin molecule recognized by autoantibodies in patients' sera and by natural autoantibodies isolated from the sera of healthy subjects. Clin Immunol Immunopathol. 1987 Oct;45(1):114-21.

2 Spencer CA. Clinical review: clinical utility of thyroglobulin antibody (TgAb) measurements for patients with differentiated thyroid cancers (DTC). J Clin Endocrinol Metab. 2011 Dec;96(12):3615-27.

3 Borowczyk M, Janicki A, Dworacki G, Szczepanek-Parulska E, Danieluk M, Barnett $J$, et al. Decreased staging of differentiated thyroid cancer in patients with chronic lymphocytic thyroiditis. J Endocrinol Invest. 2019 Jan;42(1):45-52.

4 Dvorkin S, Robenshtok E, Hirsch D, Strenov Y, Shimon I, Benbassat CA. Differentiated thyroid cancer is associated with less aggressive disease and better outcome in patients with coexisting Hashimoto thyroiditis. J Clin Endocrinol Metab. 2013 Jun;98(6):2409-14.

5 Lee JH, Kim Y, Choi JW, Kim YS. The association between papillary thyroid carcinoma and histologically proven Hashimoto's thyroiditis: a meta-analysis. Eur J Endocrinol. 2013 Feb;168(3):343-9.

6 Cunha LL, Morari EC, Guihen AC, Razolli D, Gerhard R, Nonogaki S, et al. Infiltration of a mixture of immune cells may be related to good prognosis in patients with differentiated thyroid carcinoma. Clin Endocrinol (Oxf). 2012 Dec;77(6):918-25.

7 Büyükaşık O, Hasdemir AO, Yalçın E, Celep $B$, Sengül S, Yandakçı K, et al. The association between thyroid malignancy and chronic lymphocytic thyroiditis: should it alter the surgical approach? Endokrynol Pol. 2011; 62(4):303-8.

8 Kebebew E, Treseler PA, Ituarte PH, Clark $\mathrm{OH}$. Coexisting chronic lymphocytic thyroiditis and papillary thyroid cancer revisited. World J Surg. 2001 May;25(5):632-7.

9 McLachlan SM, Feldt-Rasmussen U, Young ET, Middleton SL, Dlichert-Toft M, Siersboek-Nielsen K, et al. IgG subclass distribution of thyroid autoantibodies: a "fingerprint" of an individual's response to thyroglobulin and thyroid microsomal antigen. Clin Endocrinol (Oxf). 1987 Mar;26(3):335-46.

10 Caaveiro JM, Kiyoshi M, Tsumoto K. Structural analysis of $\mathrm{Fc}_{\mathrm{C}} / \mathrm{F}_{\mathrm{c}} \mathrm{R}$ complexes: a blueprint for antibody design. Immunol Rev. 2015 Nov;268(1):201-21.

11 Calder EA, Penhale WJ, McLeman D, Barnes EW, Irvine WJ. Lymphocyte-dependent antibody-mediated cytotoxicity in Hashimoto thyroiditis. Clin Exp Immunol. 1973 Jun; 14(2):153-8.

12 Wu X, Lun Y, Jiang H, Gang Q, Xin S, Duan $\mathrm{Z}$, et al. Coexistence of thyroglobulin antibodies and thyroid peroxidase antibodies correlates with elevated thyroid-stimulating hormone level and advanced tumor stage of papillary thyroid cancer. Endocrine. 2014 Aug; 46(3):554-60.

13 Kim ES, Lim DJ, Baek KH, Lee JM, Kim MK, Kwon HS, et al. Thyroglobulin antibody is associated with increased cancer risk in thyroid nodules. Thyroid. 2010 Aug;20(8):88591.

14 Latrofa F, Ricci D, Bottai S, Brozzi F, Chiovato L, Piaggi P, et al. Effect of Thyroglobulin Autoantibodies on the Metabolic Clearance of Serum Thyroglobulin. Thyroid. 2018 Mar; 28(3):288-94.
15 Krapp S, Mimura Y, Jefferis R, Huber R, Sondermann P. Structural analysis of human IgG-Fc glycoforms reveals a correlation between glycosylation and structural integrity. J Mol Biol. 2003 Jan;325(5):979-89.

16 Jefferis R, Lund J, Mizutani H, Nakagawa $H$, Kawazoe Y, Arata Y, et al. A comparative study of the $\mathrm{N}$-linked oligosaccharide structures of human IgG subclass proteins. Biochem J. 1990 Jun;268(3):529-37.

17 Holland M, Takada K, Okumoto T, Takahashi N, Kato K, Adu D, et al. Hypogalactosylation of serum IgG in patients with ANCAassociated systemic vasculitis. Clin Exp Immunol. 2002 Jul;129(1):183-90.

18 Arnold JN, Wormald MR, Sim RB, Rudd PM, Dwek RA. The impact of glycosylation on the biological function and structure of human immunoglobulins. Annu Rev Immunol. 2007; 25(1):21-50.

19 Jung ST, Reddy ST, Kang TH, Borrok MJ, Sandlie I, Tucker PW, et al. Aglycosylated IgG variants expressed in bacteria that selectively bind FcgammaRI potentiate tumor cell killing by monocyte-dendritic cells. Proc Natl Acad Sci USA. 2010 Jan;107(2):604-9.

20 Mimura Y, Church S, Ghirlando R, Ashton PR, Dong S, Goodall M, et al. The influence of glycosylation on the thermal stability and effector function expression of human IgG1-Fc: properties of a series of truncated glycoforms. Mol Immunol. 2000 Aug-Sep;37(12-13): 697-706.

21 Dekkers G, Treffers L, Plomp R, Bentlage AE, de Boer M, Koeleman CA, et al. Decoding the Human Immunoglobulin G-Glycan Repertoire Reveals a Spectrum of Fc-Receptor- and Complement-Mediated-Effector Activities. Front Immunol. 2017 Aug;8:877. 
22 Kelly RM, Kowle RL, Lian Z, Strifler BA, Witcher DR, Parekh BS, et al. Modulation of IgG1 immunoeffector function by glycoengineering of the GDP-fucose biosynthesis pathway. Biotechnol Bioeng. 2018 Mar;115(3):705-18.

23 Xu PC, Gou SJ, Yang XW, Cui Z, Jia XY, Chen $\mathrm{M}$, et al. Influence of variable domain glycosylation on anti-neutrophil cytoplasmic autoantibodies and anti-glomerular basement membrane autoantibodies. BMC Immunol. 2012 Mar;13(1):10.

24 Huang L, Biolsi S, Bales KR, Kuchibhotla U. Impact of variable domain glycosylation on antibody clearance: an LC/MS characterization. Anal Biochem. 2006 Feb;349(2):197207.

25 Parekh RB, Dwek RA, Sutton BJ, Fernandes DL, Leung A, Stanworth D, et al. Association of rheumatoid arthritis and primary osteoarthritis with changes in the glycosylation pattern of total serum IgG. Nature. 1985 Aug; 316(6027):452-7.

26 Theodoratou E, Thaçi K, Agakov F, Timofeeva MN, Štambuk J, Pučić-Baković M, et al. Glycosylation of plasma IgG in colorectal cancer prognosis. Sci Rep. 2016 Jun;6(1): 28098.

27 Yuan S, Li Q, Zhang Y, Huang C, Wu H, Li Y, et al. Changes in anti-thyroglobulin IgG glycosylation patterns in Hashimoto's thyroiditis patients. J Clin Endocrinol Metab. 2015 Feb;100(2):717-24.

28 Zhao L, Liu M, Gao Y, Huang Y, Lu G, Gao Y, et al. Glycosylation of sera thyroglobulin antibody in patients with thyroid diseases. Eur J Endocrinol. 2013 Mar;168(4):585-92.

29 Caturegli P, Kuppers RC, Mariotti S, Burek CL, Pinchera A, Ladenson PW, et al. IgG subclass distribution of thyroglobulin antibodies in patients with thyroid disease. Clin Exp Immunol. 1994 Dec;98(3):464-9.

30 Vidarsson G, Dekkers G, Rispens T. IgG subclasses and allotypes: from structure to effector functions. Front Immunol. 2014 Oct;5: 520.

31 Burton DR, Gregory L, Jefferis R. Aspects of the molecular structure of IgG subclasses. Monogr Allergy. 1986;19:7-35.

32 Tsuchiya N, Endo T, Shiota M, Kochibe N, Ito K, Kobata A. Distribution of glycosylation abnormality among serum IgG subclasses from patients with rheumatoid arthritis. Clin Immunol Immunopathol. 1994 Jan;70(1):47-50.

33 Perdivara I, Peddada SD, Miller FW, Tomer $\mathrm{KB}$, Deterding LJ. Mass spectrometric determination of IgG subclass-specific glycosylation profiles in siblings discordant for myositis syndromes. J Proteome Res. 2011 Jul; 10(7):2969-78.

34 Wuhrer M, Stavenhagen K, Koeleman CA, Selman MH, Harper L, Jacobs BC, et al. Skewed Fc glycosylation profiles of anti-proteinase 3 immunoglobulin G1 autoantibodies from granulomatosis with polyangiitis patients show low levels of bisection, galactosylation, and sialylation. J Proteome Res. 2015 Apr;14(4):1657-65.
35 Šimurina M, de Haan N, Vučković F, Kennedy NA, Štambuk J, Falck D, et al. Glycosylation of immunoglobulin $G$ associates with clinical features of inflammatory bowel diseases. Gastroenterology. 2018 Apr;154(5): 1320-33.e10.

36 Hao Q, Van Damme EJ, Hause B, Barre A, Chen $\mathrm{Y}$, Rougé $\mathrm{P}$, et al. Iris bulbs express type 1 and type 2 ribosome-inactivating proteins with unusual properties. Plant Physiol. 2001 Feb;125(2):866-76.

37 Raju TS. Terminal sugars of Fc glycans influence antibody effector functions of IgGs. Curr Opin Immunol. 2008 Aug;20(4):471-8.

38 Dwek RA. Biological importance of glycosylation. Dev Biol Stand. 1998;96:43-7.

39 Latrofa F, Ricci D, Montanelli L, Piaggi P, Mazzi B, Bianchi F, et al. Thyroglobulin autoantibodies switch to immunoglobulin (Ig)G1 and IgG3 subclasses and preserve their restricted epitope pattern after 131I treatment for Graves' hyperthyroidism: the activity of autoimmune disease influences subclass distribution but not epitope pattern of autoantibodies. Clin Exp Immunol. 2014 Dec;178(3): 438-46.

40 Brüggemann $\mathrm{M}$, Williams GT, Bindon $\mathrm{CI}$, Clark MR, Walker MR, Jefferis R, et al. Comparison of the effector functions of human immunoglobulins using a matched set of chimeric antibodies. J Exp Med. 1987 Nov; 166(5):1351-61.

41 Li MR, Guo XH, Gao Y, Gao YM, Lu GZ, Hui $\mathrm{Y}$, et al. Distribution and significance of IgG subclasses of serum antithyroglobulin antibody in Hashimoto thyroiditis. Zhonghua Nei Ke Za Zhi. 2008 Feb;47(2):121-4. Chinese.

42 Michaelsen TE, Aase A, Norderhaug L, Sandlie I. Antibody dependent cell-mediated cytotoxicity induced by chimeric mouse-human IgG subclasses and IgG3 antibodies with altered hinge region. Mol Immunol. 1992 Mar: 29(3):319-26.

43 Bruhns P, Iannascoli B, England P, Mancardi DA, Fernandez N, Jorieux S, et al. Specificity and affinity of human Fcgamma receptors and their polymorphic variants for human IgG subclasses. Blood. 2009 Apr;113(16): 3716-25.

44 Zhou Q, Shankara S, Roy A, Qiu H, Estes S, McVie-Wylie A, et al. Development of a simple and rapid method for producing non-fucosylated oligomannose containing antibodies with increased effector function. Biotechnol Bioeng. 2008 Feb;99(3): 652-65.

45 Rademacher TW, Williams P, Dwek RA. Agalactosyl glycoforms of IgG autoantibodies are pathogenic. Proc Natl Acad Sci USA. 1994 Jun;91(13):6123-7.

46 Shinkawa T, Nakamura K, Yamane N, ShojiHosaka E, Kanda Y, Sakurada M, et al. The absence of fucose but not the presence of galactose or bisecting $\mathrm{N}$-acetylglucosamine of human IgG1 complex-type oligosaccharides shows the critical role of enhancing antibody- dependent cellular cytotoxicity. J Biol Chem. 2003 Jan;278(5):3466-73.

47 Shields RL, Lai J, Keck R, O'Connell LY, Hong $\mathrm{K}$, Meng YG, et al. Lack of fucose on human IgG1 N-linked oligosaccharide improves binding to human Fcgamma RIII and antibody-dependent cellular toxicity. J Biol Chem. 2002 Jul;277(30):26733-40.

48 Mazziotti G, Sorvillo F, Naclerio C, Farzati A, Cioffi M, Perna R, et al. Type- 1 response in peripheral CD4+ and CD8+ T cells from patients with Hashimoto's thyroiditis. Eur J Endocrinol. 2003 Apr;148(4):383-8.

49 Ganesh BB, Bhattacharya P, Gopisetty A, Prabhakar BS. Role of cytokines in the pathogenesis and suppression of thyroid autoimmunity. J Interferon Cytokine Res. 2011 Oct; 31(10):721-31.

50 Pedro AB, Romaldini JH, Takei K. Changes of serum cytokines in hyperthyroid Graves' disease patients at diagnosis and during methimazole treatment. Neuroimmunomodulation. 2011;18(1):45-51.

51 Wang J, Balog CI, Stavenhagen K, Koeleman CA, Scherer HU, Selman MH, et al. Fc-glycosylation of IgG1 is modulated by B-cell stimuli. Mol Cell Proteomics. 2011 May; 10(5):M110.004655.

52 Fountoulakis S, Tsatsoulis A. On the pathogenesis of autoimmune thyroid disease: a unifying hypothesis. Clin Endocrinol (Oxf). 2004 Apr;60(4):397-409.

53 Ban Y, Tomer Y. The contribution of immune regulatory and thyroid specific genes to the etiology of Graves' and Hashimoto's diseases. Autoimmunity. 2003 Sep-Nov;36(6-7):36779.

54 Durante C, Tognini S, Montesano T, Orlandi F, Torlontano M, Puxeddu E, et al.; PTC Study Group. Clinical aggressiveness and long-term outcome in patients with papillary thyroid cancer and circulating anti-thyroglobulin autoantibodies. Thyroid. 2014 Jul; 24(7):1139-45.

55 Kodar K, Stadlmann J, Klaamas K, Sergeyev B, Kurtenkov O. Immunoglobulin G Fc Nglycan profiling in patients with gastric cancer by LC-ESI-MS: relation to tumor progression and survival. Glycoconj J. 2012 Jan;29(1):5766.

56 Yi CH, Weng HL, Zhou FG, Fang M, Ji J, Cheng C, et al. Elevated core-fucosylated IgG is a new marker for hepatitis B virus-related hepatocellular carcinoma. Oncoimmunology. 2015 Jul;4(12):e1011503.

57 Bones J, Byrne JC, O’Donoghue N, McManus C, Scaife C, Boissin H, et al. Glycomic and glycoproteomic analysis of serum from patients with stomach cancer reveals potential markers arising from host defense response mechanisms. J Proteome Res. 2011 Mar;10(3): 1246-65.

58 Qian Y, Wang Y, Zhang X, Zhou L, Zhang Z, $\mathrm{Xu}$ J, et al. Quantitative analysis of serum IgG galactosylation assists differential diagnosis of ovarian cancer. J Proteome Res. 2013 Sep; 12(9):4046-55. 
59 Mardente S, Lenti L, Lococo E, Consorti F, Della Rocca C, Romeo S, et al. Phenotypic and functional characterization of lymphocytes in autoimmune thyroiditis and in papillary carcinoma. Anticancer Res. May-Jun 2005; 25(3c):2483-8

60 Zivancevic-Simonovic S, Mihaljevic O, Majstorovic I, Popovic S, Markovic S, MilosevicDjordjevic O, et al. Cytokine production in patients with papillary thyroid cancer and associated autoimmune Hashimoto thyroiditis. Cancer Immunol Immunother. 2015 Aug; 64(8):1011-9.
61 Miyoshi E, Noda K, Yamaguchi Y, Inoue S, Ikeda Y, Wang W, et al. The alpha1-6-fucosyltransferase gene and its biological significance. Biochim Biophys Acta. 1999 Dec: 1473(1):9-20.

62 Ito $\mathrm{Y}$, Miyauchi A, Yoshida H, Uruno T, Nakano K, Takamura Y, et al. Expression of alpha1,6-fucosyltransferase (FUT8) in papillary carcinoma of the thyroid: its linkage to biological aggressiveness and anaplastic transformation. Cancer Lett. 2003 Oct;200(2): $167-72$.
63 Latrofa F, Ricci D, Grasso L, Vitti P, Masserini L, Basolo F, et al. Characterization of thyroglobulin epitopes in patients with autoimmune and non-autoimmune thyroid diseases using recombinant human monoclonal thyroglobulin autoantibodies. J Clin Endocrinol Metab. 2008 Feb;93(2):591-6.

64 Wuhrer M, Porcelijn L, Kapur R, Koeleman CA, Deelder A, de Haas M, et al. Regulated glycosylation patterns of IgG during alloimmune responses against human platelet antigens. J Proteome Res. 2009 Feb;8(2):450-6. 